\title{
Evaluating the induced-odour emission of a Bt maize and its attractiveness to parasitic wasps
}

\author{
Ted C.J. Turlings ${ }^{1, *}$, Philippe M. Jeanbourquin ${ }^{1}$, Matthias Held ${ }^{1} \&$ Thomas Degen ${ }^{2}$ \\ ${ }^{1}$ Laboratoire d'Ecologie Animale et Entomologie, Institut de Zoologie, Université de Neuchâtel, Case Postale 2, \\ 2007 Neuchâtel, Switzerland \\ ${ }^{2}$ Station fédérale de recherches en production végétale, Changins, 1260 Nyon, Switzerland
}

Received 29 April 2005; accepted 17 June 2005

Key words: Bacillus thuringiensis, maize, parasitoids, tritrophic interactions, Zea mays

\begin{abstract}
The current discussion on the safety of transgenic crops includes their effects on beneficial insects, such as parasitoids and predators of pest insects. One important plant trait to consider in this context is the emission of volatiles in response to herbivory. Natural enemies use the odours that result from these emissions as cues to locate their herbivorous prey and any significant change in these plant-provided signals may disrupt their search efficiency. There is a need for practical and reliable methods to evaluate transgenic crops for this and other important plant traits. Moreover, it is imperative that such evaluations are done in the context of variability for these traits among conventional genotypes of a crop. For maize and the induction of volatile emissions by caterpillar feeding this variability is known and realistic comparisons can therefore be made. Here we used a six-arm olfactometer that permits the simultaneous collection of volatiles emitted by multiple plants and testing of their attractiveness to insects. With this apparatus we measured the induced odour emissions of Bt maize (Bt11, N4640Bt) and its near-isogenic line (N4640) and the attractiveness of these odours to Cotesia marginiventris and Microplitis rufiventris, two important larval parasitoids of common lepidopteran pests. Both parasitoid species were strongly attracted to induced maize odour and neither wasp distinguished between the odours of the transgenic and the isogenic line. Also wasps that had previously experienced one of the odours during a successful oviposition divided their choices equally between the two odours. However, chemical analyses of collected odours revealed significant quantitative differences. The same 11 compounds dominated the blends of both genotypes, but the isogenic line released a larger amount of most of these. These differences may be due to altered resource allocation in the transgenic line, but it had no measurable effect on the wasps' behaviour. All compounds identified here had been previously reported for maize and the differential quantities in which they were released fall well within the range of variability observed for other maize genotypes.
\end{abstract}

\section{Introduction}

Risk assessment of the use of insect resistant transgenic plants in agriculture includes studies on

\footnotetext{
* Author for correspondence E-mail: ted.turlings@unine.ch
}

the consequences for non-target insects (Conner et al., 2003). Of particular interest are beneficial insects such as pollinators (Picard-Nizou et al., 1995; Arpaia, 1996; Poppy, 1998; Malone et al., 2001) and natural enemies of pests (Hilbeck et al., 1998; Birch et al., 1999; Bell et al., 2001), but the effects on non-target Lepidoptera has also 
received much attention (Losey et al., 1999; Jesse \& Obrycki, 2000; Wraight et al., 2000; Zangerl et al., 2001). The debate on how to conduct these assessments has not yet been settled, but it is commonly accepted that realistic tests should compare conventional methods with the strategy of employing transgenic plants, although this is rarely done as yet. Another aspect that we feel needs consideration is the comparison of any transgenically altered genotype with a representative range of existing genotypes that are conventionally used. Performance of insects on different genotypes can vary considerably, which is true for herbivores (Lewis, 1984; Fox, 1993; Preszler \& Price, 1995; Cronin \& Abrahamson, 1999) as well as their natural enemies (Fox et al., 1990; Kester \& Barbosa, 1991; Benrey et al., 1998; Turlings \& Benrey, 1998; Harvey et al., 2003). It seems therefore important to test, for instance, how reduced survival of natural enemies on a Bt maize plant compares to survival on a representative range of conventionally bred varieties.

Various studies have shown that plants respond to herbivory with the production and emission of volatile substances that are attractive to parasitoids and predators (Vet \& Dicke, 1992; Turlings \& Benrey, 1998; Dicke \& Vet, 1999; Turlings \& Wäckers, 2004). This important role of the plant in determining the success rate of host or prey location has been considered as one aspect that needs to be included in the assessment of possible changes in transgenic plants. Schuler et al. (1999, 2003) have made an important first contribution to such assessments with a study on transgenic oilseed rape that expresses a Bt (Bacillus thuringiensis) toxin as a control against the diamondback moth, Plutella xylostella. They confirmed the importance of volatiles emitted from caterpillar-damaged leaves for the host location behaviour of the parasitoid Cotesia plutellae and found that the increased feeding by $\mathrm{Bt}$ resistant diamondback larvae rendered $\mathrm{Bt}$ oilseed rape much more attractive than if susceptible larvae were feeding on the transgenic plants. These results imply that the parasitoids may help slow down the development of resistance against $\mathrm{Bt}$ in the diamondback moth (Schuler et al., 1999). As yet, no information has been obtained on the chemical composition of odours emitted by transgenic plants. It is pertinent that such assessments of transgenic plants are not only done by comparing them with their isogenic lines, but also with a broad selection of other genotypes in order to place possible changes in the context of existing variability among conventional varieties.

We have been studying the caterpillar-induced odour emissions in maize and their role as hostfinding signals for parasitoids (Turlings et al., 1990, 1995; Turlings \& Wäckers, 2004). The odour emissions are systemic and are triggered by elicitors in the oral secretions of the caterpillars. There is considerable variability among maize varieties in the quantities and relative composition of these signals (Gouinguené et al., 2001; Fritzsche-Hoballah et al., 2002). We recently tested the volatile emissions of 31 maize inbred lines that are commonly used by U.S. and European breeders to select new maize varieties for commercial use (Degen et al., 2004). These lines represent the full spectrum of genetic variability for cultivated maize from the Western hemisphere, which is reflected in the tremendous variability in odour emissions, both in terms of quantity and quality (composition of the volatile blends). Some examples are shown in Figure 1. This information on the existing variability of induced volatile emissions in maize provides a good basis with which to evaluate transgenic maize plants.

The purpose of the current study was to design a method with which to compare the odour emissions of transgenic and non-transgenic plants and to test their attractiveness to parasitoids. With the use of a six-arm olfactometer we simultaneously collected the induced volatiles emitted by Bt maize (Bt11, N4640Bt) and its isogenic line (N4640) and measured the attractiveness of the odours to the two larval endoparasitoids Cotesia marginiventris and Microplitis rufiventris. Both wasps are braconids and attack early larval stages of various lepidopteran pests, which they kill before much harm is done to a plant (FritzscheHoballah \& Turlings, 2001; Hoballah et al., 2004). These parasitoids can readily distinguish among odours of different intensity or quality, and particularly C. marginiventris prefers odours that it has experienced during an oviposition (Hoballah \& Turlings, 2005; Tamò, 2005). If transgenic and isogenic lines differ in the odours they emit, this should be reflected in the responses of the wasps. To enhance their responsiveness and their ability to distinguish among odours we also tested females of both species that had previously 


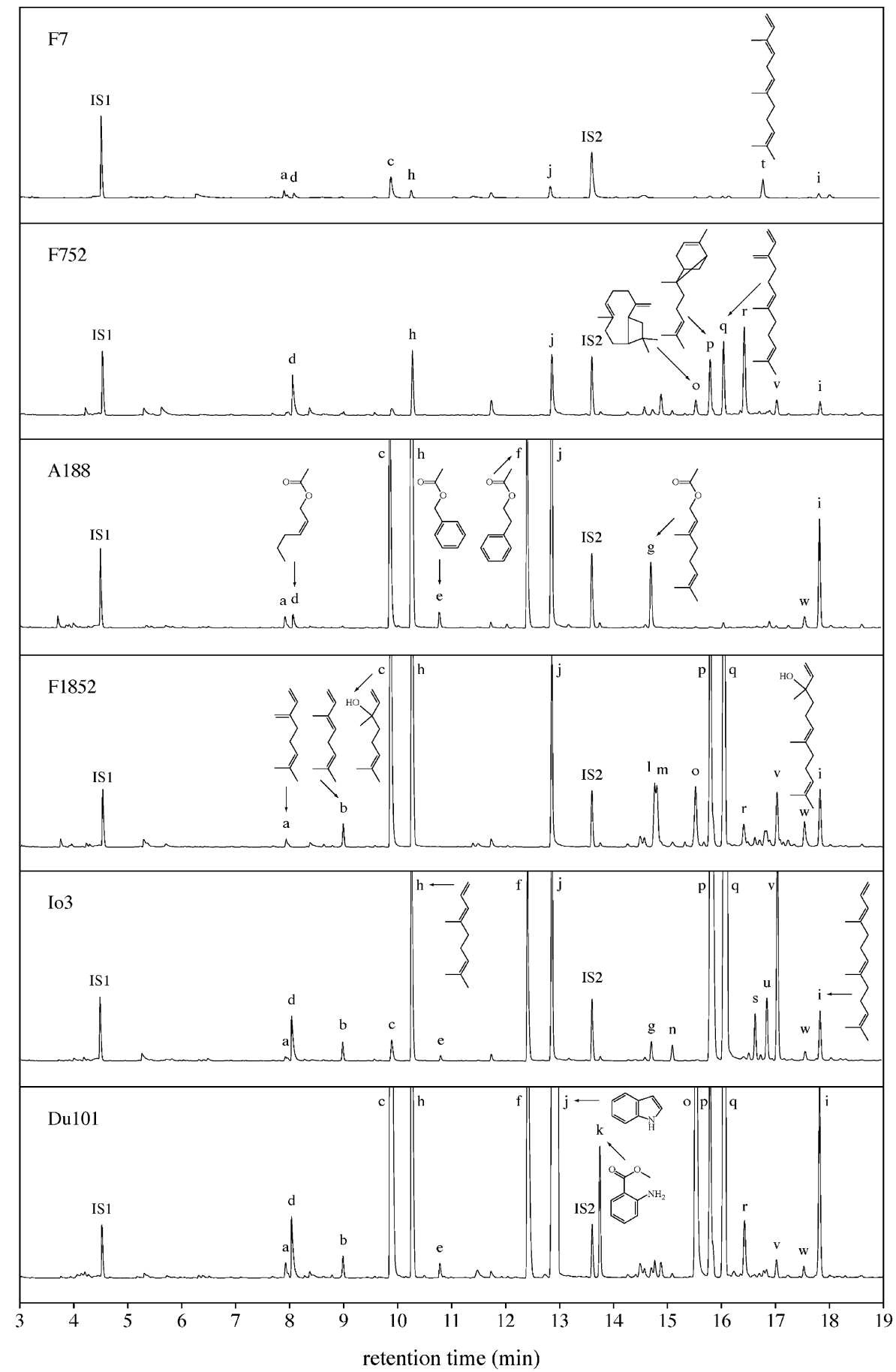

Figure 1. Typical chromatographic profiles of herbivore-induced volatiles emitted by six maize inbred lines (F7, F752, A188, F1852, Io3 and Du101), representative for the variability that can be observed in these emissions. The volatiles were collected $8 \mathrm{~h}$ after the plants had been injected with the regurgitant of Spodoptera littoralis caterpillars (for details on the inbred lines and treatment see Degen et al., 2004). The represented compounds are: a. $\beta$-myrcene; b. (E)- $\beta$-ocimene; c. linalool; d. ( $Z$ )-3-hexen-1-yl acetate; e. benzyl acetate; f. phenethyl acetate; g. geranyl acetate; h. (3E)-4,8-dimethyl-1,3,7-nonatriene; i. (3E,7E)-4,8,12- trimethyl1,3,7,11-tridecatetraene; j. 1- $H$-indole; k. methyl anthranilate; 1 . cyclosisosativene; m. $\alpha$-ylangene; n. unknown; o. $(E)$ - $\beta$-caryophyllene; p. $(E)$ - $\alpha$-bergamotene; q. $(E)$ - $\beta$-farnesene; r. germacrene-D; s. unknown; t. $(E, E)$ - $\alpha$-farnesene; u. $\beta$-bisabolene; v. unknown; w. (E)-nerolidol. IS1 and IS2 are the internal standards $n$-octane and nonyl-acetate, respectively. 
experienced the odour of either plant during an oviposition in a larva of Spodoptera littoralis, a suitable host.

\section{Materials and methods}

\section{Maize plants}

For this study we evaluated the maize hybrids Novartis N4640Bt (Yield-Gard, Cry1Ab, event $\mathrm{Bt} 11)$ and its non-Bt isogenic equivalent N4640. Plants were grown from seeds, which were individually planted in plastic pots $(6-\mathrm{cm}$ high, 8-cm diameter) with fertilised commercial soil (Coop, Switzerland). The pots were placed in a climate chamber $\left(23^{\circ} \mathrm{C}, 60 \%\right.$ r.h., and LD 16:8 h, $50,000 \mathrm{~lm} / \mathrm{m}^{2}$ ). Eight days after planting, the plants that would serve as odour sources in the olfactometer were transplanted into a glass pot $(11-\mathrm{cm}$ high 5 -cm internal diameter) with a $50-55-\mathrm{mm}$ ground glass male connector that could be inserted into an odour source vessel of the olfactometer. The plants were induced to produce odours (see below) on that same day and used in bio-assays the following morning. Plants that were to be used to precondition the wasps were left in their original pots, but received the same treatment.

\section{Induction of plants}

Between $17.00 \mathrm{~h}$ and $18.00 \mathrm{~h}$ the day before each assay, plants were induced to emit volatiles by scratching two leaves $\left(2-\mathrm{cm}^{2}\right)$ with a scalpel and applying $10 \mu \mathrm{l}$ S. littoralis regurgitant to the damaged sites. Regurgitant was collected and applied as described in Turlings et al. (1993). After treatment the plants were placed in the odour sources vessels of the olfactometer (see below) and remained there until and throughout the assays.

This artificial, but effective method of induction was chosen because of repeatability and to ensure equal treatment of both hybrid lines. The alternative of having Spodoptera larvae feed on the plants would have resulted in considerable less feeding damage on the Bt line than on the non-Bt line, which would certainly reflect in differential volatile emissions. The purpose of this study was to evaluate if transgenesis has caused changes in the plant's capacity to produce inducible odour emissions.

\section{The insects}

For all bio-assays, we used 2-3 day-old females of the solitary endoparasitoids Cotesia marginiventris and Microplitis rufiventris. The rearing colony of C. marginiventris originated from the USDAARS, Biological Control and Mass Rearing Research Unit (Mississippi, U.S.A.). The colony of $M$. rufiventris was started and replenished with individuals reared from Spodoptera littoralis larvae that had been collected in crop fields near Alexandria, Egypt. S. littoralis larvae used for rearing were provided by Syngenta (Stein, Switzerland). See Fritzsche-Hoballah \& Turlings (2001) for details on the rearing procedures.

\section{Pre-conditioning of the wasps}

Before releasing them in the olfactometer, female wasps received one of three possible experiences; (1) they were left naïve, meaning that they never contacted hosts or plant material as adults (NAÏVE); (2) they were trained with the odour of Bt maize by having them oviposit twice in second instar S. littoralis larvae that had been placed on induced Bt seedlings (BT experience); or (3) they were trained with the odour of isogenic maize by having them oviposit twice in second instar $S$. littoralis larvae that had been placed on induced isogenic seedlings (ISO experience).

Experiencing an odour during an oviposition is known to increase the responsiveness of parasitoids to the experienced odour as a result of associative learning (Turlings et al., 1993; Vet et al., 1995). The olfactometer was used in a previous study to test the learning ability of the two parasitoid species and it was found that C. marginiventris exhibits a preference for an experienced odour, whereas M. rufiventris shows an overall increase in responsiveness, but no change in preference (Tamò, 2005). It can be expected that if the wasps perceive differences between the odours of the two genotypes that at least C. marginiventris females will show an increased attraction to the odour with which they had a positive encounter. 


\section{The olfactometer}

The olfactometer has been described in detail by Turlings et al. (2004). It is a device in which wasps are exposed to six different airflows that enter a central glass exposure chamber through six arms. Each flow can potentially carry a different odour. In this study only two of the flows (from opposite arms) carried an odour (one of Bt maize and one of isogenic maize), while the other flows served as controls and carried only humidified pure air. The olfactometer has been shown to be highly effective in measuring the relative attractiveness of induced plant odours for both parasitoids (Turlings et al., 2004; Tamò, 2005). During the assays, $50 \%$ of the airflow was pulled through trapping filters (Heath \& Manukian, 1992) for $3 \mathrm{~h}$ to collect volatiles as described by Turlings et al. (2004) for later analyses (see below).

\section{Bio-assays}

On a given day, 3 groups of 6 wasps were released in the olfactometer: one group of naïve wasps, one group trained on Bt maize and one groups trained on isogenic maize. Treatment groups were alternated and ordered differently on different days. For each parasitoid species this experiment was replicated on 12 different days, which ensured that all possible position for the opposing odour sources were tested.

The experienced wasps received their experience just before they were placed in the olfactometer. Each group of six was given 30 min to make a choice, after which all choices were recorded and wasps were removed with an aspirator. The wasps that had stayed in the central exposure chamber were counted as "no choice".

\section{Analyses of volatiles}

Immediately after each experiment, the volatiles collected on the trapping filters were extracted with $150 \mu \mathrm{l}$ of methylene chloride and two internal standards (n-octane and nonyl acetate, each $200 \mathrm{ng}$ in $10 \mu \mathrm{l}$ methylene chloride) were added to these samples. For the analysis, an aliquot of $3 \mu 1$ was injected on-column with the use of an automated injection system onto an apolar HP-1 capillary column $(30 \mathrm{~m}, 0.25-\mathrm{mm}$ I.D., $0.25 \mu \mathrm{m}$ film thickness), which was preceded by a deactivated retention gap (10-m, 0.25-mm I.D., Connex, U.S.A.) and a deactivated precolumn (30-cm, 0.53-mm I.D., Connex, U.S.A.). The columns were housed in a Hewlett Packard model HP 6890 gas chromatograph equipped with a flame ionisation detector. The oven was held at $50^{\circ} \mathrm{C}$ for $3 \mathrm{~min}$ and then programmed at $8^{\circ} \mathrm{C} / \mathrm{min}$ to $230^{\circ} \mathrm{C}$, where it was maintained for $9.5 \mathrm{~min}$. Helium $(24-\mathrm{cm} / \mathrm{s})$ was used as carrier gas. HP GC Chemstation software was used to quantify all major components based on the known quantity of internal standards. Initial identification of most compounds was based on comparisons of retention times with those from previous studies (Bernasconi et al., 1998; Turlings \& Benrey, 1998) and was confirmed with the mass spectrometry analysis of some samples. For this purpose $3 \mu \mathrm{l}$ aliquot were injected in pulsed splitless mode into a GC, using the same column and temperature programme (Agilent 5973, transfer line $230^{\circ} \mathrm{C}$, source $230^{\circ} \mathrm{C}$, quadrupole $150^{\circ} \mathrm{C}$, ionisation potential $70 \mathrm{eV}$, scan range $30-240 \mathrm{amu}$ ). Two sesquitepenes did not separate well from each other and could only be tentatively identified as (+)cycloisosativene and ylangene. For practical purposes they were quantified together. Total quantities of volatiles were calculated based on their peak areas as compared to those of the internal standards.

\section{Statistical evaluation of the data}

Analyses of the wasp choice data were based on log-linear models (Davison et al., 2003; Section 10.7) fitted for the expected distribution of the wasps within the olfactometer (Turlings et al., 2004). The results for each test day were used as a replication (12 replicates). Only those insects that made a choice were included in the analyses of the results (over $80 \%$ of the wasps). Wasp choices consisted of counts (N1-N6), where $N_{i}$ denotes the number of wasps observed to choose arm $i$ and the assumption is that these counts follow a multinomial distribution with probabilities (p1-p6). Here we used a $\log$ linear form $\log \left\{E\left(N_{i j}\right)\right\}=y_{j}+x_{i}$ whereby the frequencies of choice $N_{i j}$ of the treatment $i$ for the $j$ th replication is taken to depend linearly on a covariate $\exp \left(x_{i}\right)$, which measures the relative attractiveness for that treatment. The model was fitted by maximum quasilikelihood estimation in the software package R (http://stat.ethz.ch/CRAN/), and its relative 
adequacy was assessed through likelihood ratio statistics and examination of residuals.

We tested for quantitative differences in the total odour emissions as well as for the emissions for individual compounds. For this a paired $t$-test was used, comparing the plants tested on a particular day as pairs $(n=23)$. In case of non-normal distribution, a Wilcoxon signed rank test was performed.

\section{Results}

\section{Odour emissions}

Eleven compounds were found to consistently dominate the blends collected from the transgenic as well as the isogenic line (Figure 2; Table 1). These same compounds are commonly released by many other maize varieties in response to caterpillar feeding (Figure 1; Gouinguené et al., 2001; Fritzsche-Hoballah et al., 2002; Degen et al., 2004). The ratios among the compounds are also common and did not differ between the isogenic and transgenic line. On average the isogenic plants released significantly more in terms of total amounts (Figure 2(b); paired $t$-test, $P=0.003$ ). Of the individual compounds, linalool, $(3 E)-4,8-$ dimethyl-1,3,7-nonatriene, phenethyl acetate,
(E)- $\alpha$-bergamotene, $(E)$ - $\beta$-farnesene, $(E)$-nerolidol, and $(3 E, 7 E)-4,8,12$-trimethyl-1,3,7,11-tridecatetraene, were released in significantly smaller amounts by the transgenic line than by the isogenic line (Table 1). For average quantities of the remaining compounds ( $Z$ )-3-hexenyl acetate, "(+)-cycloisosativene + ylangene", $\beta$-caryophyllene, and $\beta$-sesquiphellandrene no differences were found between the two maize lines.

\section{Parasitoid responses}

Neither C. marginiventris nor $M$. rufiventris distinguished among the odours of the two maize lines tested. Both parasitoid species were strongly attracted to the odours of Spodoptera-damaged maize plants (Figure 3), with about $80 \%$ of C. marginiventris and about $70 \%$ of $M$. rufiventris females choosing an arm with a plant odour. However, in none of the cases was there a significant difference in the choices between the two lines. This was true for naïve and experienced wasps and no shift in preference was observed if they experienced an oviposition on a transgenic or an isogenic plant. Table 2 shows the statistical analyses of the data, demonstrating that there is no effect of maize line or experience on the choices
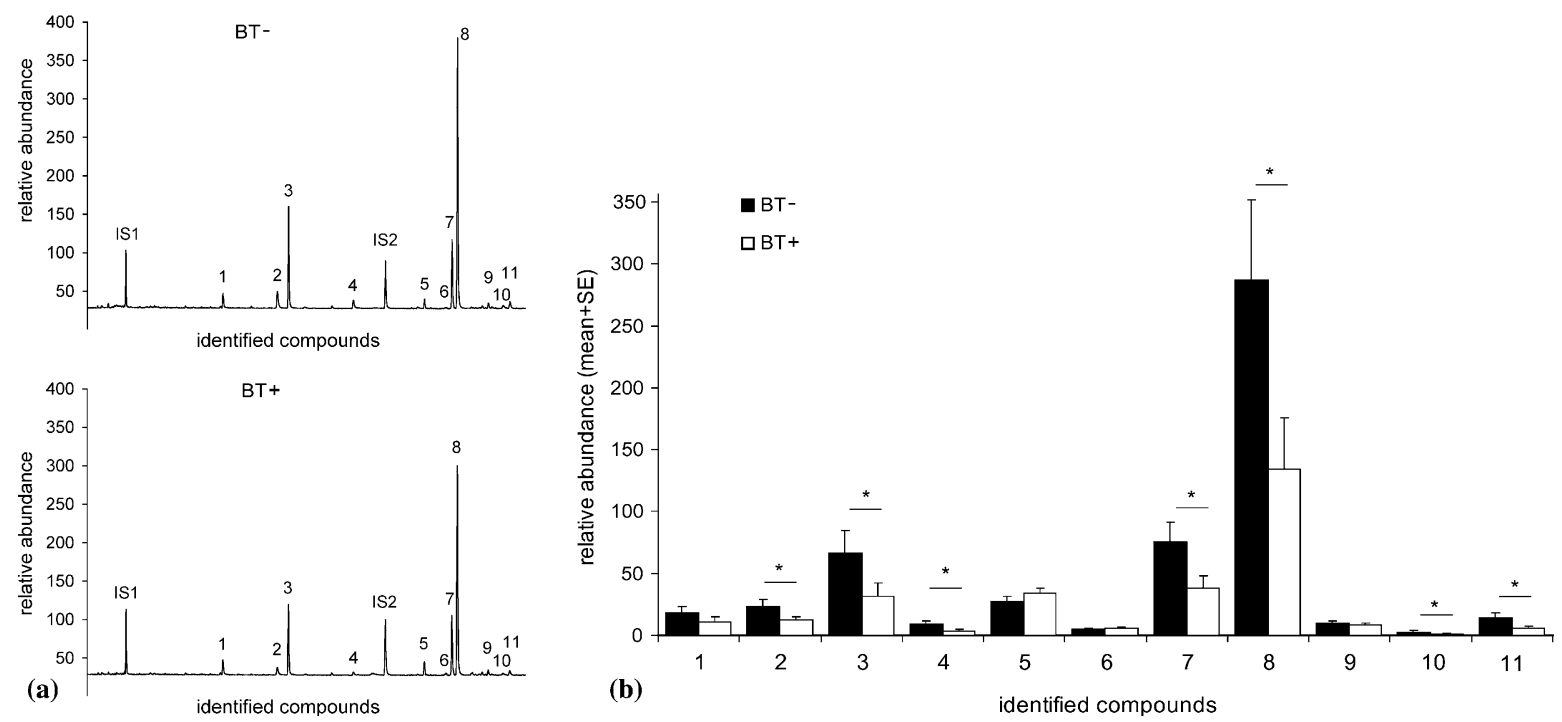

Figure 2. (a). Typical chromatographic profiles of herbivore-induced volatiles emitted by non-Bt (BT-) and Bt (BT + ) maize plants. The labelled peaks are: 1. (Z)-3-hexenyl acetate; 2. linalool; 3. (3E)-4,8-dimethyl-1,3,7-nonatriene; 4. phenethyl acetate; 5. " $(+)$-cycloisosativene $+\alpha$-ylangene"; 6 . $\beta$-caryophyllene; 7. $(E)$ - $\beta$-farnesene; 8 . (E)- $\alpha$-bergamotene; $9 . \quad \beta$-sesquiphellandrene; 10 . $(E)$-nerolidol; 11. (3E,7E)-4,8,12-trimethyl-1,3,7,11-tridecatetraene. IS1 and IS2 are the internal standards $n$-octane and nonyl-acetate, respectively. (b) Relative amounts were estimated by comparing peak areas with those of the internal standards $(n=23)$. Compound numbers correspond with the labels in Figure 2(a). The asterisks indicate significant differences between non-Bt and Bt plants. 
Table 1. Test statistics for the comparisons of volatile concentrations of $\mathrm{Bt}+$ vs. $\mathrm{Bt}-$ plants

\begin{tabular}{|c|c|c|c|c|}
\hline Compound & Statistic & df & $t$-value & $P$ \\
\hline (Z)-3-hexenyl acetate & Paired $t$-test & 22 & 1.964 & 0.062 \\
\hline Linalool & Paired $t$-test & 22 & 2.891 & 0.008 \\
\hline (3E)-4,8-dimethyl-1,3,7-nonatriene & Paired $t$-test & 22 & 3.114 & 0.005 \\
\hline Phenethyl acetate & Wilcoxon-signed-rank & & & 0.016 \\
\hline$«(+)$-cycloisosativene $+a$-ylangene» & Paired $t$-test & 22 & -1.585 & 0.127 \\
\hline$b$-caryophyllene & Paired $t$-test & 22 & -1.19 & 0.247 \\
\hline$(E)$-b-farnesene & Paired $t$-test & 22 & 3.732 & 0.001 \\
\hline (E)-a-bergamotene & Paired $t$-test & 22 & 3.31 & 0.003 \\
\hline$b$-sesquiphellandrene & Paired $t$-test & 22 & 1.978 & 0.061 \\
\hline$(E)$-nerolidol & Wilcoxon-signed-rank & & & 0.018 \\
\hline$(3 E, 7 E)-4,8,12$-trimethyl-1,3,7,11-tridecatetraene & Paired $t$-test & 22 & 3.046 & 0.006 \\
\hline All compounds & Paired $t$-test & 22 & 3.393 & 0.003 \\
\hline
\end{tabular}

Bold $P$-values indicate a significant difference. The numbers correspond to the labels used in Figure 2.

made by the wasps. From several other studies (Turlings et al., 1993), including some with these two species in the six-arm olfactometer (Tamò, 2005), we know that shifts in preference do occur if the wasps distinguish between the odours that they are offered. This is apparently not the case here.

\section{Discussion}

The odour collections showed an almost two-fold overall reduction in the volatile emissions in the transgenic maize line. For the individual compounds the reduction was significant for seven of the eleven principal compounds. However, the differences in the emissions were not reflected in the responses by the C.marginiventris and $M$. rufiventris. The two parasitoids were strongly attracted to induced plants, but in none of the tests did they distinguish between the odour of the transgenic and the isogenic line, even if they had an oviposition experience on one of the lines. Such experiences are known to lead to associative learning, which increases the responsiveness of parasitoids to the odours they perceive during oviposition (Turlings et al., 1993; Vet et al., 1995). In previous studies (Turlings et al., 2004; Tamò, 2005) we found that the wasps are capable of distinguishing between different odours in the same olfactometer and they make a clear choice, which can be reinforced by experience. Here the oviposition experiences slightly increased the overall attraction to the plant odours, but had no effect on the wasps' choices between the two lines. The wasps divided their choices between the two odours equally, suggesting that they did not perceive any differences.

The consequences of introducing a foreign gene into a crop plant for the attraction of parasitoids has been previously studied for a system comprising $\mathrm{Bt}$ oilseed rape, the diamondback moth and the parasitoid Cotesia plutellae (Schuler et al., 1999). On Bt oilseed rape the larvae feed far less, which results in a strong reduction in volatile emissions. This is not the case for resistant larvae, which fed just as readily on Bt rape as on nontransgenic rape (Schuler et al., 1999). Because of the direct effect of the Bt also on the feeding rate of $S$. littoralis (personal observations) we opted for a standardized artificial induction of the plants by scratching leaves and applying regurgitant. This latter treatment causes comparable emissions in maize and in attraction of parasitoids (Turlings et al., 1990). Obviously, the presented results do not reflect what would happen in terms of parasitoid attraction in the field if the Bt maize were to be introduced. On Bt maize the caterpillars' feeding rate would be strongly reduced, which should have consequences for parasitoid attraction. As in the Schuler et al. (1999) study, it can be expected that resistant herbivores will feed considerably more than non-resistant ones, resulting in comparably more volatiles and increased parasitoid attraction.

Key to the current study is that we have ample information on the range of variability in odour 
Cotesia marginiventris

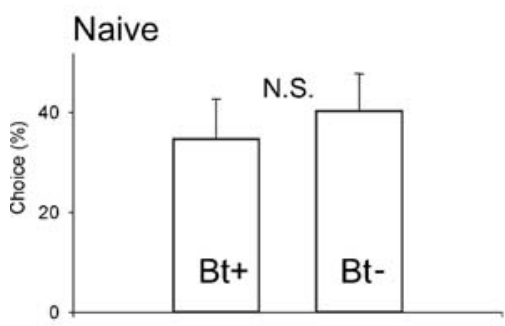

Experienced with $\mathrm{Bt}+$
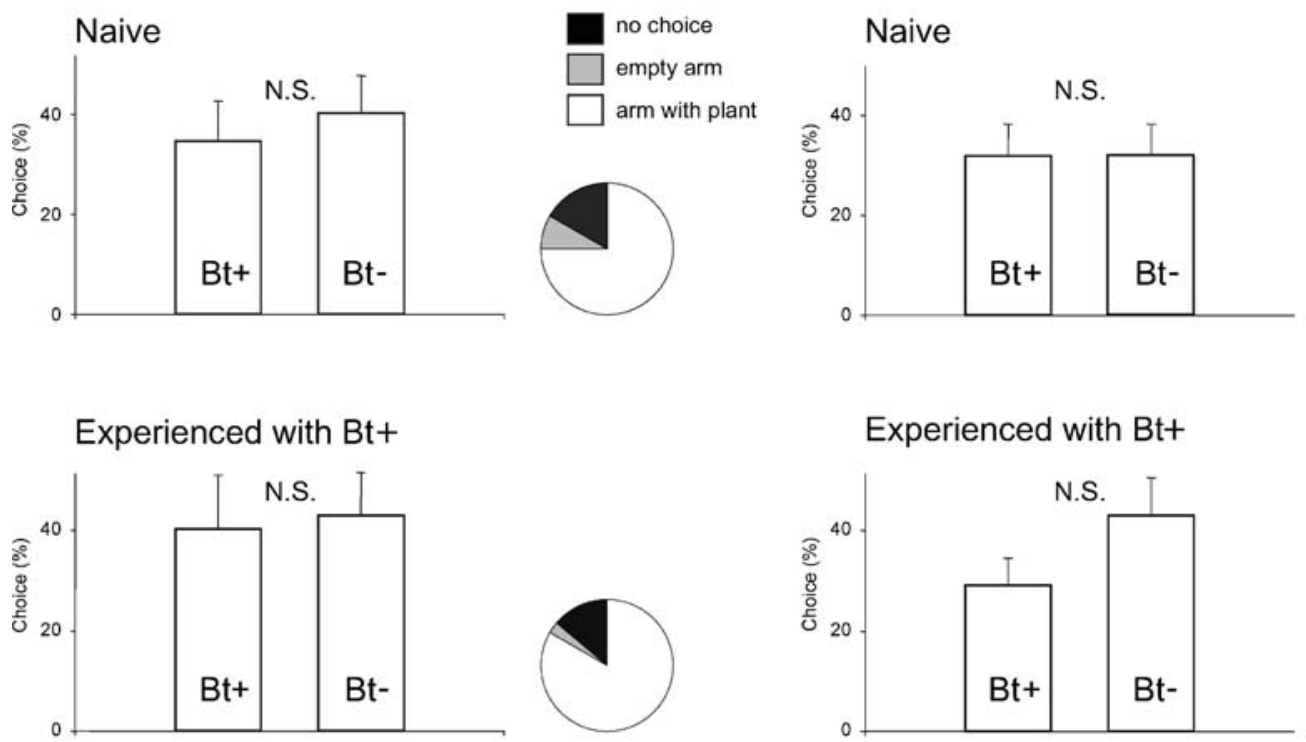

no choice

$\square$ empty arm arm with plant

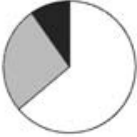

\section{Experienced with $\mathrm{Bt}+$}
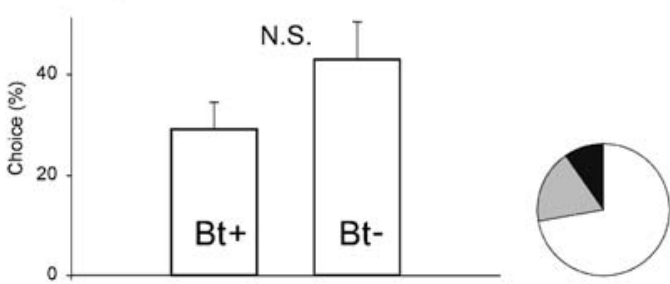

Experienced with Bt-
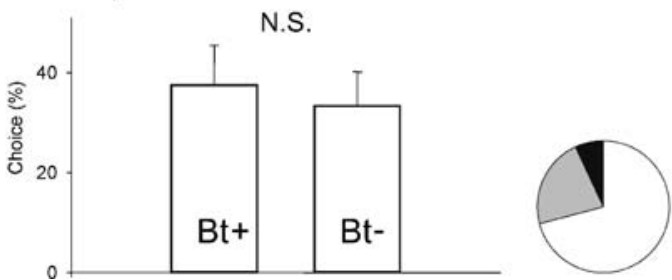

Figure 3. Responses of females of the parasitoids Cotesia marginiventris and Microplitis rufiventris to the odours of induced $\mathrm{Bt}$ and non-Bt plants in a 6-arm olfactometer. The wasps were either naïve or experienced with the odour of one of the two induced genotypes. The bars represent the percentage of the responding females that chose for one of the two plant odours, whereas the pie-graphs show the proportions of females that stayed in the centre of the olfactometer (no choice), walked into an empty arm or walked into an arm with a plant odour. For each of the six experiments $12 \times 6$ wasps were tested.

Table 2. Test statistics for wasp choice behaviour based on a log-linear model as explained in the text

\begin{tabular}{lll}
\hline Statistics & Cotesia marginiventris & Microplitis rufiventris \\
\hline \multicolumn{2}{l}{ Bt treatment } & \\
df & 70 & 70 \\
$F$ & 0.9008 & 0.3434 \\
$\operatorname{Pr}(>F)$ & 0.3459 (n.s.) & 0.5598 (n.s.) \\
Experience & & \\
df & 68 & 68 \\
$F$ & 0.1293 & 0.2197 \\
$\operatorname{Pr}(>F)$ & 0.8789 (n.s.) & 0.8033 (n.s.)
\end{tabular}

emissions among different maize lines (Gouinguené et al., 2001; Fritzsche-Hoballah et al., 2002; Degen et al., 2004). Variability among maize lines is tremendous and it can be concluded that both maize lines tested here produce a volatile blend that falls well within the common range. This is true for the total amount produced (quantity), as well as for the composition of the blend (quality) (compare Figures 1 and 2).

Although the emissions by the transgenic line can be considered normal when compared to the overall emissions by conventional lines, it is significantly different from its isogenic line. This 
may be due to a differential resource allocation in the transgenic line. The production of the Bt toxin might cost plant resources that could otherwise be invested in secondary compounds such as the volatiles sampled here. In the current study this did not result in reduced attraction of beneficial parasitoids, but other effects, such as reduced production of common maize defence compounds could have consequences for the vulnerability of the plant to pests.

To our knowledge this is the first study showing that the introduction of a Bacillus thuringiensis gene in maize has resulted in a reduction of caterpillar-induced odour emissions. Yet, the quantities of the individual compounds produced by the transgenic line are within the range of variability that can be found among conventional maize lines and the attraction of two common parasitoids of lepidopteran maize pests was found not to differ between the transgenic and its isogenic line. It is recommended that further studies into the consequences of genetic transformation of crop plants also employ approaches that compare the studied traits of transgenic plants with a representative range of conventional varieties.

\section{Acknowledgements}

We thank the members of the group entomologie évolutive (University of Neuchâtel), as well as the group of Franz Bigler at Agroscope, Reckenholz, Switzerland for their continuous support and scientific feedback. Statistical advice was provided by Ingrid Ricard and Anthony Davison (EPFLausanne). This research was funded by the Swiss National Center of Competence in Research "Plant Survival”.

\section{References}

Arpaia S (1996) Ecological impact of Bt-transgenic plants: 1. Assessing possible effects of CryIIIB toxin on honey bee (Apis mellifera L.) colonies. J Genet Breed 50: 315-319.

Bell HA, Fitches EC, Marris GC, Bell J, Edwards JP, Gatehouse JA et al. (2001) Transgenic GNA expressing potato plants augment the beneficial biocontrol of Lacanobia oleracea (Lepidoptera; Noctuidae) by the parasitoid Eulophus pennicornis (Hymenoptera; eulophidae). Trans Res 10: 35-42.
Benrey B, Callejas A, Rios L, Oyama K and Denno RF (1998) The effects of domestication of Brassica and Phaseolus on the interaction between phytophagous insects and parasitoids. Biol Control 11: 130-140.

Bernasconi ML, Turlings TCJ, Ambrosetti L, Bassetti P and Dorn S (1998) Herbivore-induced emissions of maize volatiles repel the corn leaf aphid, Rhopalosiphum maidis. Entomol Exp Appl 87: 133-142

Birch ANE, Geoghegan IE, Majerus MEN, McNicol JW, Hackett CA, Gatehouse AMR et al. (1999) Tri-trophic interactions involving pest aphids, predatory 2 -spot ladybirds and transgenic potatoes expressing snowdrop lectin for aphid resistance. Mol Breeding 5: 75-83.

Conner AJ, Glare TR and Nap JP (2003) The release of genetically modified crops into the environment - Part II. Overview of ecological risk assessment. Plant J 33: 19-46.

Cronin JT and Abrahamson WG (1999) Host-plant genotype and of her herbivores influence goldenrod stem galler preference and performance. Oecologia 121: 392-404.

Davison AC, Hinkley DV and Young GA (2003) Recent developments in bootstrap methodology. Stat Sci 18: 141-157.

Degen T, Dillmann C, Marion-Poll F and Turlings TCJ (2004) High genetic variability of herbivore-induced volatile emission within a broad range of maize inbred lines. Plant Physiol 135: 1928-1938.

Dicke M and Vet LEM (1999) Plant-carnivore interactions: evolutionary and ecological consequences for plant, herbivore and carnivore. In: Herbivores Between Plants and Predators. pp 483-520..

Fox CW (1993) A quantitative genetic analysis of oviposition preference and larval performance on 2 hosts in the bruchid beetle, Callosobruchus maculatus. Evolution 47: 166-175.

Fox LR, Letourneau DK, Eisenbach $\mathbf{J}$ and Vannouhuys S (1990) Parasitism rates and sex ratios of a parasitoid wasp effects of herbivore and plant quality. Oecologia 83: 414 419.

Fritzsche-Hoballah ME, Tamò C and Turlings TCJ (2002) Differential attractiveness of induced odors emitted by eight maize varieties for the parasitoid Cotesia marginiventris: is quality or quantity important? J Chem Ecol 28: 951-968.

Fritzsche-Hoballah ME and Turlings TCJ (2001) Experimental evidence that plants under caterpillar attack may benefit from attracting parasitoids. Evol Ecol Res 3: 553-565.

Gouinguené S, Degen T and Turlings TCJ (2001) Variability in herbivore-induced odour emissions among maize cultivars and their wild ancestors (teosinte). Chemoecology 11: 9-16.

Harvey JA, van Dam NM and Gols R (2003) Interactions over four trophic levels: foodplant quality affects development of a hyperparasitoid as mediated through a herbivore and its primary parasitoid. J Anim Ecol 72: 520-531.

Heath RR and Manukian A (1992) Development and evaluation of systems to collect volatile semiochemicals from insects and plants using a charcoal-infused medium for air purification. J Chem Ecol 18: 1209-1226.

Hilbeck A, Baumgartner M, Fried PM and Bigler F (1998) Effects of transgenic Bacillus thuringiensis corn-fed prey on mortality and development time of immature Chrysoperla carnea (Neuroptera: Chrysopidae). Environ Entomol 27: 480-487.

Hoballah ME, Degen T, Bergvinson D, Savidan A, Tamò C and Turlings TCJ (2004) Occurrence and direct control potential of parasitoids and predators of the fall armyworm (Lepidoptera: Noctuidae) on maize in the subtropical lowlands of Mexico. Agric For Entomol 6: 83-88. 
Hoballah ME and Turlings TCJ (2005) The role of fresh versus old leaf damage in the attraction of parasitic wasps to herbivore-induced maize volatiles. $J$ Chem Ecol (in press).

Jesse LCH and Obrycki JJ (2000) Field deposition of Bt transgenic corn pollen: lethal effects on the monarch butterfly. Oecologia 125: 241-248.

Kester KM and Barbosa P (1991) Postemergence learning in the insect parasitoid, Cotesia congregata (Say) (Hymenoptera, Braconidae). J Insect Behav 4: 727-742.

Lewis AC (1984) Plant quality and grasshopper feeding - effects of sunflower condition on preference and performance in Melanoplus differentialis. Ecology 65: 836-843.

Losey JE, Rayor LS and Carter ME (1999) Transgenic pollen harms monarch larvae. Nature 399: 214-214.

Malone LA, Burgess EPJ, Gatehouse HS, Voisey CR, Tregida EL and Philip BA (2001) Effects of ingestion of a Bacillus thuringiensis toxin and a trypsin inhibitor on honey bee flight activity and longevity. Apidologie 32: 57-68.

Picard-Nizou AL, Pham-Delegue MH, Kerguelen V, Douault P, Marilleau R, Olsen L et al. (1995) Foraging behaviour of honey bees (Apis mellifera L.) on transgenic oilseed rape (Brassica napus L. var. oleifera). Trans Res 4: 270-274.

Poppy G (1998) Transgenic plants and bees: the beginning of the end or a new opportunity? Bee World 79: 161-164.

Preszler RW and Price PW (1995) A test of plant-vigor, plantstress, and plant-genotype effects on leaf-miner oviposition and performance. Oikos 74: 485-492.

Schuler TH, Potting RPJ, Denholm I, Clark SJ, Clark AJ, Stewart CN et al. (2003) Tritrophic choice experiments with Bt plants, the diamondback moth (Plutella xylostella) and the parasitoid Cotesia plutellae. Trans Res 12: 351-361.

Schuler TH, Potting RPJ, Denholm I and Poppy GM (1999) Parasitoid behaviour and Bt plants. Nature 400: 825-826.

Tamò C (2005) A comparative study on the plant odour preferences and learning ability of three solitary endoparasitoids of Spodoptera species. In: Dissertation. University of Neuchâtel.
Turlings TCJ and Benrey B (1998) Effects of plant metabolites on the behavior and development of parasitic wasps. Ecoscience 5: 321-333.

Turlings TCJ, Davison AC and Tamò C (2004) A six-arm olfactometer permitting simultaneous observation of insect attraction and odour trapping. Physiol Entomol 29: 45-55.

Turlings TCJ, Loughrin JH, McCall PJ, Rose USR, Lewis WJ and Tumlinson JH (1995) How caterpillar-damaged plants protect themselves by attracting parasitic wasps. Proc Natl Acad Sci USA 92: 4169-4174.

Turlings TCJ, McCall PJ, Alborn HT and Tumlinson JH (1993) An elicitor in caterpillar oral secretions that induces corn seedlings to emit chemical signals attractive to parasitic wasps. $J$ Chem Ecol 19: 411-425.

Turlings TCJ, Tumlinson JH and Lewis WJ (1990) Exploitation of herbivore-induced plant odors by host-seeking parasitic wasps. Science 250: 1251-1253.

Turlings TCJ and Wäckers F (2004) Recruitment of predators and parasitoids by herbivore-injured plants. In: Cardé RT and Millar JG (eds), Advances in Insect Chemical Ecology Cambridge University Press.

Vet LEM and Dicke M (1992) Ecology of infochemical use by natural enemies in a tritrophic context. Proc Natl Acad Sci USA 37: 141-172.

Vet LEM, Lewis WJ and Cardé RT (1995) Parasitoid foraging and learning. In: Cardé RT and Bell WJ (eds), Chemical Ecology of Insects 2. (pp. 65-101) Chapman \& Hall, New York.

Wraight CL, Zangerl AR, Carroll MJ and Berenbaum MR (2000) Absence of toxicity of Bacillus thuringiensis pollen to black swallowtails under field conditions. Proc Natl Acad Sci USA 97: 7700-7703.

Zangerl AR, McKenna D, Wraight CL, Carroll M, Ficarello P, Warner R et al. (2001) Effects of exposure to event 176 Bacillus thuringiensis corn pollen on monarch and black swallowtail caterpillars under field conditions. Proc Natl Acad Sci USA 98: 11,908-11,912. 